\title{
A linguagem probabilística de alunos do quinto ano do ensino fundamental: identificando fenômenos ou experimentos aleatórios
}

\section{The probabilistic language of fifth grade elementary school students: identifying random phenomena or experiments}

\author{
Ailton Paulo de Oliveira Junior ${ }^{1 *}$, Fátima Aparecida Kian ${ }^{2}$
}

\begin{abstract}
RESUMO
Consideramos que o estudo de conceitos probabilísticos nos anos iniciais é essencial à formação da criança, visto que no mundo atual recebemos uma grande quantidade de informações, necessitando compreender fenômenos aleatórios ou não aleatórios. Os elementos linguísticos no processo de ensino e aprendizagem de probabilidade é entendida como uma linguagem especializada, sendo corroborado pela Base Nacional Comum Curricular - BNCC ao ser indicado que é essencial a formação de conceitos de natureza probabilística desde os anos iniciais do Ensino Fundamental. Portanto, neste estudo nos concentramos em mostrar o processo de construção da linguagem utilizada para conceituar fenômenos ou experimentos aleatórios utilizados por alunos do quinto ano do Ensino Fundamental de uma escola municipal de Barueri, São Paulo. Para isso, realizamos um estudo exploratório com alunos que receberam algum tipo de instrução prévia sobre o assunto, especificamente, a multiplicidade de termos (expressões escritas). Foram realizadas análises textuais por meio do software IRaMuTeQ na qual utilizamos análises multivariadas (Classificação Hierárquica Descendente - CHD). Os resultados mostram a predominância de palavras e expressões verbais da língua comum relacionado principalmente ao significado intuitivo de probabilidade.
\end{abstract}

Palavras-Chave: Linguagem probabilística; Experimento ou fenômeno aleatório; Anos iniciais do Ensino Fundamental; Análise Textual Multivariada.

\begin{abstract}
We consider that the study of probabilistic concepts in the early years is essential for the formation of the child, since in the current world we receive a large amount of information, even needing to understand random or non-random phenomena. The linguistic elements in the teaching and learning process of probability is understood as a specialized language, being corroborated by the National Common Curricular Base - BNCC when it is indicated that the formation of concepts of a probabilistic nature from the early years of Elementary School is essential. . Therefore, in this study we focus on showing the process of construction of the language used to conceptualize phenomena or random experiments used by students of the fifth year of Elementary School in a municipal school in Barueri, São Paulo. For this, we carried out an exploratory study with students who received some kind of previous instruction on the subject, specifically, the multiplicity of terms (written expressions). Textual analyzes were performed using the IRaMuTeQ software in which we used multivariate analyzes (Descending Hierarchical Classification - CHD). The results show the predominance of common language words and verbal expressions related mainly to the intuitive meaning of probability.
\end{abstract}

\footnotetext{
${ }^{1}$ Universidade Federal do ABC. Doutor em Educação. ailton.junior@ufabc.edu.br

${ }^{2}$ Universidade Federal do ABC. Doutoranda em Ensino e História das Ciências e da Matemática.

fatima.kian@ufabc.edu.br
} 
Keywords: Probabilistic language; Random experiment or phenomenon; Initial years of Elementary School; Multivariate Textual Analysis.

\section{INTRODUÇÃO}

Buscamos nesse trabalho descrever e analisar como emergem elementos linguísticos probabilísticos de alunos do quinto ano do Ensino Fundamental, no caso o conceito de fenômeno ou experimento aleatório, entendida como uma linguagem especializada e apoiada na Base Nacional Comum Curricular - BNCC (BRASIL, 2018).

A BNCC (BRASIL, 2018) indica que o estudo da probabilidade nos anos iniciais do Ensino Fundamental tem como finalidade promover a compreensão de que nem todos os eventos são determinísticos, devendo ser considerado de forma gradativa.

Além disso, a BNCC alerta que émuito comum que pessoas julguem impossíveis eventos que nunca viram acontecer. Dessa forma, nessa fase, é importante que os alunos verbalizem, em eventos que envolvem o acaso, os resultados que poderiam ter acontecido em oposição ao que realmente aconteceu (BRASIL, 2018).

\section{ASPECTOS REFERENTES À LINGUAGEM PROBABILÍSTICA: EXPERIMENTOS OU FENÔMENOS ALEATÓRIOS}

O conceito de aleatório nem sempre se mostra claro, porque é referente a uma entidade abstrata, não inteiramente definida, aumentando as dificuldades potenciais para os alunos. A aleatoriedade é um objeto multifacetado, conforme mostrado em várias interpretações recebidas ao longo da história (BATANERO; HENRY; PARZYSZ, 2005; SALDANHA; LIU, 2014).

Batanero (2015) lembra que o conceito de aleatoriedade não é simples e que ao longo da história teve diferentes significados, estando associado a discussões filosóficas, podendo ser encontrado diferentes definições. É geralmente definido por meio de algumas propriedades como "imprevisibilidade", "possibilidade de vários resultados", "incontrolável", dentre outros.

Morgado et al. (2004) expressa que fenômenos ou experimentos aleatórios acontecem constantemente em nossa vida diária e que repetidos sob as mesmas condições, produzem resultados geralmente diferentes. 
Especificamente em relação aos fenômenos ou experimentos aleatórios, Salmerón (2015) considera que é necessário realizar observações sobre o que acontece em determinados momentos, a fim de identificar os possíveis resultados e poder concluir se um resultado é mais previsível do que os outros.

Ainda nos apoiamos em Vásquez (2018) que apresenta um conjunto de situações em que o aluno possa identificar situações do cotidiano e, então, que possa esse indicar se o experimento ou fenômeno apresentado se configura como aleatório.

Concebemos que como os alunos já receberam instrução anterior sobre esse assunto, devem conceber situações que se configuram como um fenômeno aleatório. Além disso, consideramos que os estudantes devem relacionar o acaso com a sorte e com termos relacionados a fenômenos que ocorrem inesperadamente, por acaso.

A atividade aqui elaborada considera os conteúdos propostos na BNCC para os anos iniciais do Ensino Fundamental, Brasil (2018), de forma a possibilitar aos alunos a compreensão, inicialmente, de conceitos básicos de probabilidade, nesse momento a noção de acaso, identificando fenômenos ou experimentos aleatórios.

Portanto, nessa parte do trabalho é apresentado situação para que os alunos possam distinguir os fenômenos aleatórios, solicitando esse escreva uma palavra ou palavras sobre a possibilidade da situação proposta ocorrer.

Oliveira Júnior et al. (2019), elaboraram problemas abordando a identificação de experimentos aleatórios associados à BNCC (BRASIL, 2018), que fazem parte de um jogo pedagógico manipulável com base na Teoria Antropológica do Didático - TAD, composto por situações problema ou tarefas, constituída de uma sequência de subtarefas, que podem ser realizadas utilizando diversas técnicas justificadas pela tecnologia que se utiliza de teorias relacionadas à Probabilidade como objeto de estudo.

Em relação à linguagem probabilística, Gal (2005) propõe cinco aspectos para a aquisição dessa linguagem (verbal, numérica, tabular, gráfica e simbólica), entendida como especializada para comunicar o acaso, sendo desenvolvida como uma progressão de idéias probabilísticas para obter entendimento adequado da Teoria da Probabilidade.

Neste estudo, focaremos especificamente na linguagem verbal (refere-se à diversidade de termos e expressões verbais), apoiando-se no Conselho Nacional de Professores de Matemática - NCTM dos Estados Unidos, NCTM (2003), ao considerar o papel fundamental no processo de ensino e aprendizagem da probabilidade nas primeiras idades, devido à estreita relação entre expressões comuns e linguagem probabilística. 
Dentro do tipo de linguagem verbal, segundo Shuard e Rothery (1984), é possível distinguir três categorias de expressões utilizadas no ensino da matemática e que aqui associaremos ao ensino de probabilidade: (1) Expressões verbais específicas da probabilidade - as que não fazem parte da linguagem comum, ou seja, correspondem a uma linguagem técnica da probabilidade; (2) Expressões verbais ligadas à probabilidade - usadas tanto no contexto probabilístico quanto no cotidiano, mas nem sempre têm o mesmo significado nos dois contextos; (3) Expressões verbais comuns: têm significados iguais ou muito próximos tanto no contexto probabilístico quanto na vida cotidiana.

Além disso, D’Amore (2007) indica que a linguagem com a qual se faz matemática possui um código semiológico próprio, acarretando várias convenções mais ou menos explícitas: o uso de escritas específicas, as expressões simbólicas (fórmulas ou representações numéricas). Assim, não só os símbolos matemáticos, mas a própria linguagem natural, parece bem mais complexa, uma vez que com poucas palavras são dadas muitas informações.

\section{MÉTODO}

A seguir apresentamos os procedimentos metodológicos que utilizamos para a realização da coleta de dados, registros e análises que convergem para discussões e considerações sobre o ensino de probabilidade nos anos iniciais do Ensino Fundamental.

\subsection{Tipo de estudo}

Esta pesquisa é do tipo exploratório, de abordagem qualitativa e quantitativa por meio de questionário disponibilizado pelo Google Forms e analisado pelo software IRaMuTeQ (Interface R para Texto Multidimensional e Análise de Questionário) para descrever e analisar como emergem elementos linguísticos probabilísticos de alunos do quinto ano do Ensino Fundamental, o conceito de fenômeno ou experimento aleatório.

Assim, para a análise dos dados, utilizamos o software IRaMuTeQ, que foi desenvolvido como ferramenta auxiliar do processo de codificação dos elementos trazidos por meio da coleta de dados (MUTOMBO, 2013). É um software gratuito e com fonte aberta, desenvolvido por Pierre Ratinaud (LAHLOU, 2012; MARCHAND; RATINAUD, 2012) e licenciado por GNU GPL (v2), que permite fazer análises estatísticas sobre corpus textuais e sobre tabelas indivíduos/palavras, ancorando-se no software R e na linguagem Python. 
O referido software foi utilizado para realizar uma análise lexical quantitativa que considera a palavra como unidade, também oferecendo a sua contextualização no corpus ou no instrumento de pesquisa ou questionário. Cada pergunta do instrumento é composta por conteúdos semânticos, formando o banco de dados ou corpus analisado pelo software.

\subsection{Amostra}

Para obter os dados, o instrumento foi enviado aos 167 alunos matriculados no quinto ano do Ensino Fundamental, distribuídos dentre cinco turmas de uma escola pública no município de Barueri, São Paulo, sendo que 61 (36,5\%) alunos responderam.

O estudo envolveu estudantes que receberam algum tipo de instrução prévia sobre conceitos básicos de probabilidade. A pesquisa foi submetida e aprovada pelo comitê de ética em pesquisa da Plataforma Brasil da Universidade Federal do ABC - UFABC sob o número CAAE: 42350720.7.0000.5594.

\subsection{Procedimentos Metodológicos}

Para alcançar a análise indicada no item referente às análises textuais, é necessário ter ferramentas que permitam a análise microscópica das práticas probabilísticas colocadas em jogo na resolução da tarefa proposta que buscarão identificar e explicar a multiplicidade de termos, expressões orais e escritas, símbolos e representações utilizados na conceituação de fenômenos ou experimentos aleatórios.

Assim, para a análise dos resultados obtidos, utilizamos a análise textual, um tipo específico de análise de dados, na qual tratamos de material verbal transcrito, ou seja, de textos (NASCIMENTO-SCHULZE; CAMARGO, 2000). Tem várias finalidades, sendo possível analisar textos, entrevistas, documentos, redações etc. No caso desse estudo, as respostas indicadas pelos alunos ao instrumento disponibilizado via Google Forms.

Assim, por meio da análise textual é possível descrever um material produzido, seja individual ou coletivamente, como também pode-se utilizar a análise textual com a finalidade relacional, comparando produções diferentes em função de variáveis específicas que descrevem quem produziu o texto.

Considerando Nascimento-Schulze e Camargo (2000), tendo como base a compreensão da análise textual, descrevemos a seguir conceitos importantes e já associados ao contexto desse trabalho (Quadro 1). 
Quadro 1: Conceitos importante para a análise textual.

\begin{tabular}{|c|l|}
\hline Conceito & \multicolumn{1}{|c|}{ Descrição do conceito } \\
\hline Corpus & $\begin{array}{l}\text { Construído pelo pesquisador, é o conjunto texto que se pretende analisar. No } \\
\text { caso dessa trabalho, por exemplo, um corpus será composto das respostas a } \\
\text { questões abertas, que faz parte de um questionário empregado como } \\
\text { instrumento de uma pesquisa do tipo enquete. }\end{array}$ \\
\hline Textos & $\begin{array}{l}\text { Em se tratando de respostas a questões abertas de um questionário, cada texto } \\
\text { será composto da adição dos trechos obtidos das respostas somente quando elas } \\
\text { se referirem a um mesmo tema (uma mesma questão ou pergunta). Caso as } \\
\text { questões referiram-se a temas ou aspectos diferentes, é necessário realizar uma } \\
\text { análise para cada questão. Como mencionado anteriormente, a análise é } \\
\text { sensível à estruturação do estímulo que produz o material textual, e isto é uma } \\
\text { importante fonte de invalidação das conclusões. Quando as respostas } \\
\text { apresentarem uma média em torno de três à cinco linhas, é necessário um } \\
\text { número bem maior de respostas para a constituição de um corpus de análise. }\end{array}$ \\
\hline Segmentos & $\begin{array}{l}\text { São excertos de texto, na maior parte das vezes, do tamanho de até três linhas, } \\
\text { dimensionadas pelo próprio software em função do tamanho do corpus. Os } \\
\text { de texto } \\
\text { segmentos de textos são considerados o ambiente das palavras. }\end{array}$ \\
\hline
\end{tabular}

Fonte: Elaborado pelos autores segundo Nascimento-Schulze e Camargo (2000).

A Figura 1 indica a relação sequencial dos conceitos que se fazem importantes para a realização de uma análise textual.

Figura 1: Noções de corpus, texto, segmento de texto.

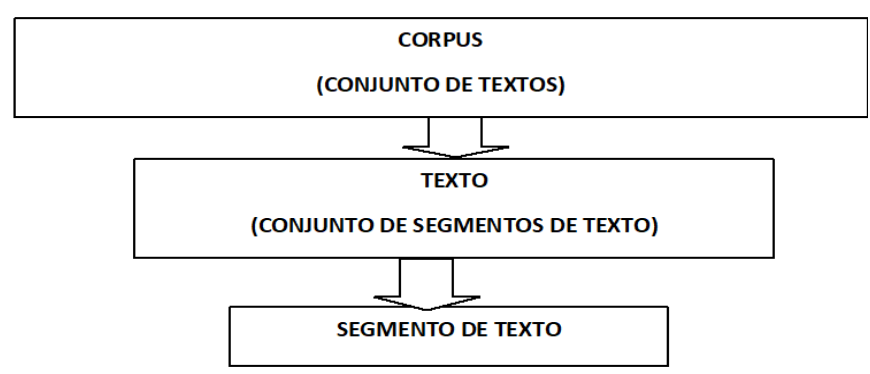

Fonte: Elaborado pelos autores.

A definição das unidades de análise é realizada pelo pesquisador e dependerá da natureza da pesquisa, ou seja, as respostas do instrumento dizem respeito aos "n" participantes de uma questão aberta, portanto, "n" textos.

$\mathrm{O}$ conjunto de textos constitui-se um corpus de análise. $\mathrm{O}$ corpus adequado à análise do tipo Classificação Hierárquica Descendente - CHD, deve constituir-se num conjunto textual centrado em um tema. O material textual deve ser monotemático, pois a análise de textos sobre vários itens previamente estruturados ou diversos temas resulta na reprodução da estruturação prévia dos mesmos (CAMARGO, 2005).

Destacamos que as análises do tipo CHD, para serem úteis à classificação de qualquer material textual, requerem uma retenção mínima de $75 \%$ dos segmentos de texto, sendo que para alguns autores há de se considerar a possibilidade de se considerar o aproveitamento de 70\% dos segmentos de textos (CAMARGO; JUSTO, 2013). 
Portanto, o trabalho utilizou de forma conjugada um software desenvolvido para a análise textual e de um procedimento de análise de conteúdo clássica. O software IRaMuTeQ apoia-se em cálculos efetuados sobre a co-ocorrência de palavras em segmentos de texto, buscando distinguir classes de palavras que representem formas distintas de discurso sobre o tópico de interesse da investigação.

\section{IDENTIFICAÇÃO DE FENÔMENOS OU EXPERIMENTOS}

\section{ALEATÓRIOS PELOS ALUNOS DO QUINTO ANO}

Os chamados fenômenos ou experimentos aleatórios ${ }^{3}$ são processos reais ou hipotéticos nos quais os possíveis resultados podem ser identificados antecipadamente, mas não podemos prever com certeza o que vai acontecer, pois o acaso representa um papel preponderante, possuindo duas características fundamentais: (1) O resultado não pode ser previsto com certeza; (2) Embora não seja possível afirmar que resultado em particular ocorrerá, é possível descrever o conjunto de todos os resultados possíveis do experimento. Assim, apresentamos uma das situações apresentadas aos alunos como fenômeno ou experimento aleatório Figura 2.

Figura 2: Situação associada a um fenômeno ou experimento aleatório.

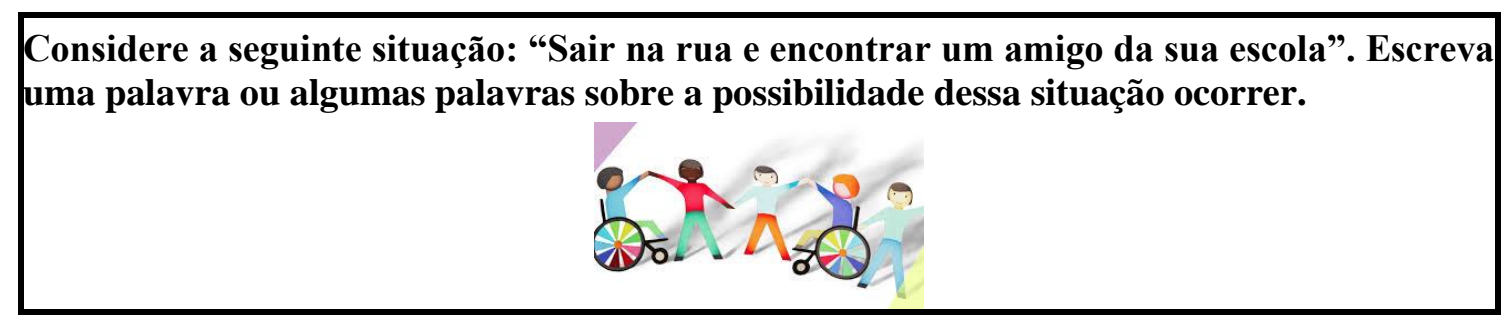

Fonte: Elaborado pelos autores.

No resumo das respostas de 60 alunos (98,4\% do total), podemos observar que as palavras de maior frequência, ou aquelas que apresentam o número de formas ativas com frequência maior ou igual a 3 são indicadas na Tabela 1.

Lembramos que as formas ativas e suplementares são as palavras consideradas ativas (adjetivos, nomes, verbos e advérbios) e suplementares (artigos e pronomes), exceto os artigos e as preposições que foram eliminados.

\footnotetext{
${ }^{3}$ http://clubes.obmep.org.br/blog/sala-de-estudo-probabilidades/
} 
Tabela 1: Indicação das formas ativas com três ou mais indicações na análise referente às respostas dos alunos à primeira questão que apresenta um fenômeno ou experimento aleatório.

\begin{tabular}{c|c||c|c}
\hline Palavra (função lexical) & Frequência & Palavra (função lexical) & Frequência \\
\hline Bem (advérbio) & 9 & Estar (verbo) & 4 \\
\hline Amigo (nome) & 9 & Chance (nome) & 3 \\
\hline Acontecer (verbo) & 9 & Bairro (nome) & 3 \\
\hline Não (advérbio) & 7 & Mesmo (adjetivo) & 3 \\
\hline Possível (adjetivo) & 6 & Achar (verbo) & 3 \\
\hline Provável (adjetivo) & 6 & Certeza (nome) & 3 \\
\hline Encontrar (verbo) & 5 & Coincidência (nome) & 3 \\
\hline Morar (verbo) & 4 & Ocorrer (verbo) & 3 \\
\hline Rua (nome) & 4 & \multicolumn{2}{|l}{}
\end{tabular}

Fonte: Organizado a partir da saída do IRaMuTeQ.

Observamos na Tabela 1 que a palavra com maior frequência é o advérbio "Bem”, sendo que pelo dicionário online Antônio Houaiss ${ }^{4}$, seu significado voltado à aspectos probabilísticos é aquilo que é realizado como certeza, ou provavelmente, ou ainda aquilo que é adequado, exato, quanto à natureza da coisa que se fala.

Identificamos ainda que o verbo "Acontecer" se refere a processo que está diretamente relacionado com o significado dado pelos alunos à noção de acaso, ratificado pelo sistema periférico da análise de similitude, no qual, contém as seguintes palavras: acaso, algo, motivo, coisa, esperar, nada e imprevisível. Outras duas palavras merecem ser destacadas que são: 1) O adjetivo "Possível", ter alguma chance de se realizar, embora não saiba quando e se ocorrerá; 2) O adjetivo "Provável" que reflete algo esperável ou que provavelmente acontecerá ou ainda com grande possibilidade para que aconteça.

Para iniciar a apresentação do que os alunos indicaram referente às respostas à questão em análise que apresenta um fenômeno ou experimento aleatório, utilizamos o método de Reinert que propõe uma CHD segundo o método descrito por Reinert (1990), visando obter classes de segmentos de texto (ST) que, ao mesmo tempo, apresentam vocabulário semelhante entre si e vocabulário diferente das ST das outras classes.

Enfatizamos que a escolha pela utilização de uma ou outra técnica de análise depende das características do problema e dos objetivos da pesquisa (LEBLANC, 2015). Nessa direção, o referencial teórico-metodológico do pesquisador, acrescido do suporte de softwares de análise lexicométrica, podem conferir maior confiabilidade às inferências realizadas em pesquisas qualitativas (CAMARGO; JUSTO, 2014; SANTOS et al., 2017).

\footnotetext{
${ }^{4}$ https://houaiss.uol.com.br/corporativo/apps/uol_www/v5-4/html/index.php\#0
} 
Portanto, esta análise é baseada na proximidade léxica e na ideia de que palavras usadas em contexto similar que estão associadas ao mesmo mundo léxico e são parte de mundos mentais específicos ou sistemas de representação. Nessa análise, os segmentos de texto são classificados de acordo com seu respectivo vocabulário e o conjunto de termos é particionado de acordo com a frequência das raízes das palavras. O sistema procura obter classes formadas por palavras que são significantemente associadas com aquela classe (a significância começa com o teste de quiquadrado $-\chi^{2}$ ).

Segundo Oliveira (2015) o teste de quiquadrado é uma das análises mais importantes do IRaMuTeQ, pois, o software utiliza a lógica de correlação, partindo de segmentações do corpus textual, juntamente com a lista de formas reduzidas e o dicionário (no caso em português) disponibilizado no mesmo para apresentar um esquema hierárquico de classes. Dessa forma é processado o texto de modo que possam ser identificadas classes de vocabulário, sendo possível inferir quais ideias o corpus textual deseja transmitir, ou seja, a análise é feita a partir de uma lógica estatística processada por computador e aplicada de forma lexical.

Assim, na Figura 3 apresentamos o dendrograma gerado na CHD, que indica as partições que foram feitas no corpus até que se chegasse às duas classes finais.

Figura 3: Resultado da Classificação pelo Método de Reinert referente às respostas dos alunos à questão que apresenta um fenômeno ou experimento aleatório: Dendrograma.

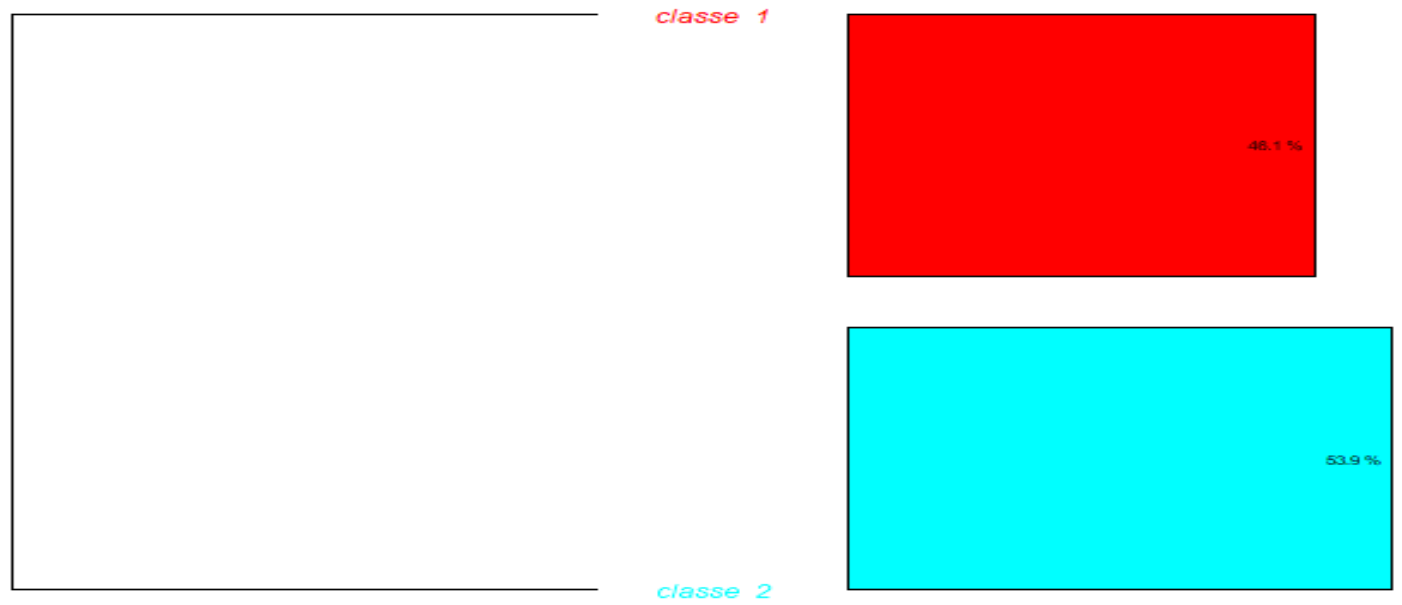

Fonte: Saída do software IRaMuTeQ.

Assim, no resultado da Classificação pelo Método de Reinert: Dendrograma, Figura 3, o corpus "Corpo" foi dividido em dois subcorpus, sendo que a classe 1 representa $46,1 \%$ do corpus total e a classe 2 representa 53,9\%.

Portanto, as duas classes contêm as formas ativas ou palavras organizadas que apresentaram maior frequência, em ordem decrescente, e que são significativas para 
representar cada um dos subcorpus por meio do teste de associação qui-quadrado, ou seja, a maior aderência delas na classe e entre as classes e que pode ser observado (Tabela 2).

Tabela 2: Indicação das formas ativas com três ou mais indicações que formam as duas classes na análise referente às respostas dos alunos à questão que apresenta um fenômeno ou experimento aleatório.

\begin{tabular}{c|c||c|c}
\hline Classe 1 & Frequência & Classe 2 & Frequência \\
\hline Amigo (Nome) & 9 & Bem (Advérbio) & 9 \\
\hline Acontecer (Verbo) & 9 & Provável (Adjetivo) & 6 \\
\hline Não (Advérbio) & 7 & & \\
\hline Encontrar (Verbo) & 5 & & \\
\hline Rua (Nome) & 4 & &
\end{tabular}

Fonte: Organizado a partir da saída do IRaMuTeQ.

Tomando o dendrograma (Figura 3) e as palavras de maior frequência e que apresentaram relação significativa entre elas (Tabela 2), buscamos identificar as respostas dos alunos à questão que apresenta um fenômeno ou experimento aleatório.

Assim, na Classe 1 que denominamos por "Apresentar situações em que vivenciaram ou poderia vivenciar a situação proposta", tomando as combinações das palavras que apresentam relação significativa, destacamos os seguintes enxertos:

$* * * * * n \_02 * \mathrm{Id} \_2 * \mathrm{Gen} \_1 * \mathrm{GM} \_1$

Já aconteceu alguns dias eu falo um oi ele para um lado e eu para o outro ou nós conversamos.

$* * * * * \mathrm{n} \_06 * \mathrm{Id} \_1 * \mathrm{Gen} \_1 * \mathrm{GM} \_1$

Você pode encontrar um amigo da sua escola, se ele estiver no parque, na praça ou no mercado.

$* * * * * \mathrm{n} \_10 * \mathrm{Id} \_1 * \mathrm{Gen} \_1 * \mathrm{GM} \_1$

Pode acontecer. Já aconteceu comigo.

$* * * * * \mathrm{n} \_20 * \mathrm{Id} \_3 * \mathrm{Gen} \_2 * \mathrm{GM} \_1$

Coincidência eu encontrar meu amigo na rua.

$* * * * * n \_22 * \mathrm{Id} \_3 * \mathrm{Gen} \_2 * \mathrm{GM} \_1$

Pode acontecer, mas não tem muita probabilidade.

*****n_25 *Id_2 *Gen_2 *GM_1

Cinquenta por cento, pois todos os meus amigos moram no mesmo bairro que eu.

$* * * * * \mathrm{n} \_45 * \mathrm{Id} \_3 * \mathrm{Gen} \_2 * \mathrm{GM} \_1$

Eu já encontrei vários amigos na rua, a possibilidade de isso acontecer novamente é bem alta. $* * * * * n \_47 * I d \_3 *$ Gen_1 *GM_1

Não achar ninguém, achar crianças que você não conheça ou nem ir pra rua.

$* * * * * \mathrm{n} \_53 * \mathrm{Id} \_2 * \mathrm{Gen} \_1 * \mathrm{GM} \_1$

Acho que isso raramente vai acontecer.

$* * * * * n \_56 * \mathrm{Id} \_2 * \mathrm{Gen} \_2 * \mathrm{GM} \_1$

Muita chance de isso acontecer. 
Na Classe 2 na qual denominamos por "Utilizar termos probabilísticos para explicar a situação proposta", tomamos as combinações das palavras que apresentam relação significativa, destacando os seguintes enxertos:

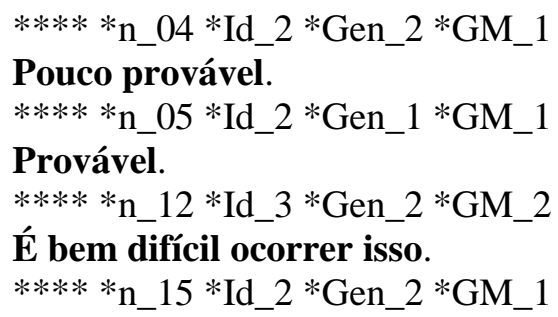

Bem difícil dessa situação ocorrer.

Para entendermos e analisarmos foi preciso identificar como foi feita a construção do discurso que compõem o corpus do texto, e assim identificarmos a estrutura inicial que relaciona as formas lexicais e o contexto presente na base de dados.

Portanto, trazemos Marocci e Nacarato (2013) quando destaca que é necessário inicialmente compreender o que os alunos entendem sobre os termos mais frequentes, para depois ampliar os significados já construídos e progredir na formação de conceitos probabilísticos, pressupondo processos de comunicação e reflexão pelos alunos.

Esse estudo converge para Vásquez e Alsina (2017) que concluíram que os significados surgem de experiências cotidianas referente ao vocabulário que compõem a linguagem probabilística e todo desenvolvimento do raciocínio probabilístico, através de expressões que denotam seus graus de crenças sobre o que pode ocorrer em um evento.

Ainda indicando exemplos para explicar a ocorrência do evento em questão, para Alveal, Levicoy e Vásquez (2018) o desenvolvimento de experimentos aleatórios dão lugar a descrever a probabilidade a partir do grau de possibilidade de ocorrência de eventos que são oriundos de diversas situações do cotidiano. 
Por fim, Marocci e Nacarato (2013), expressam que o sentido atribuído a expressões ou palavras a situações do cotidiano é subjetivo, ou seja, a compreensão de certa situação envolvendo a probabilidade será diferente para cada pessoa.

A esse respeito, Konold (1991) indica que os alunos possuem uma compreensão coerente, proveniente do contato com circunstâncias que envolvem incerteza e, a partir disso, procuram dar sentido às situações vivenciadas.

Com isso percebemos em nossos estudo que as situações diárias provocam na criança a utilização de linguagem probabilística apresentando indícios do entendimento sobre conceitos probabilísticos.

\section{CONSIDERAÇÕES FINAIS}

Buscamos nesse trabalho descrever e analisar como emergem elementos linguísticos no processo de ensino e aprendizagem de probabilidade de alunos do quinto ano do Ensino Fundamental de uma escola pública do município de Barueri, São Paulo.

Assim, o estudo em questão sugere que esses alunos do quinto ano do Ensino Fundamental possuem conhecimentos prévios e experiências do contexto cotidiano que possibilitam estudar a probabilidade desde as primeiras idades.

Segundo Bryant e Nunes (2012) a compreensão da probabilidade de resultados incertos desempenha um papel extremamente importante em nossas vidas. Dependemos disso para decidir sobre o tratamento médico que devemos seguir, o carro que compramos e as precauções que devemos tomar para proteger nossas famílias e nossos lares. Todas essas e outras decisões dependem do nosso conhecimento dos possíveis eventos que podem acontecer e nossa compreensão da probabilidade desses eventos serem diferentes.

Portanto, partimos da premissa de que para o estudo de probabilidade sejam desenvolvidas as primeiras noções e elementos de aproximação para a aquisição e desenvolvimento da linguagem probabilística. Sendo os conceitos probabilísticos complexos e com alto grau de abstração, faz-se necessário iniciar apresenta-los de forma gradual para que o aluno tenha um entendimento adequado da linguagem específica.

O estudo apontou, apoiado em Vásquez (2014), que a aprendizagem da probabilidade deve ser focada em situações cotidianas em que os conceitos de experimentos aleatórios estejam presentes. Percebeu-se que os alunos quando 
responderam à questão proposta consideraram o experimento ou fenômeno aleatório que foi apresentado, indicando que situações reais facilitam a apreensão desse conceito.

Os resultados do estudo também convergem para o que Vásquez (2018) indica ao dizer que a linguagem probabilística nas primeira idades e consequentemente nos anos iniciais do Ensino Fundamental tem ligação muito próxima com a linguagem cotidiana, pois, os primeiros elementos linguísticos fazem parte do idioma dos alunos.

Esse estudo confirma que é importante desenvolver atividades que utilizem a representação de conceitos probabilísticos, pois, permitem que o aluno identifique as situações reais propostas e, gradualmente, associá-las à linguagem probabilística.

Dessa forma, desde os anos iniciais, o aluno deve ter contato com a linguagem probabilística, partindo de aspetos mais intuitivos e naturais e, progressivamente, partir de problemas mais simples aos mais elaborados, e construir um conhecimento mais formal e sólido referente à Teoria da Probabilidade.

\section{REFERENCIAS}

ALVEAL, F.; LEVICOY, D.; VÁSQUEZ, C. Evaluación de la alfabetización probabilística del profesorado en formación y en activo. Revista Estudios Pedagógicos, Valdivia-Chile, v. 44, n. 1, p. 135-156, 2018.

BATANERO, C. Understanding randomness: challenges for research and teaching. In: Conferência CERME 9, 9., 2015. Anais... Congress of European Research in Mathematics Education. Praga, Fevereiro, 2015.

BATANERO, C.; HENRY, M.; PARZYSZ, B. The nature of chance and probability. In: JONES, G. (Ed.). Exploring probability in school: challenges for teaching and learning. New York: Springer, 2005. p. 15-37.

BRASIL. Base Nacional Comum Curricular: Educação é a base. Ministério da Educação, Brasília, dez. 2018. 600 p. Disponível em:

$<$ http://basenacionalcomum.mec.gov.br/images/BNCC_EI_EF_110518_versaofinal_site .pdf> Acesso em: 16 jan. 2022.

BRYANT, P.; NUNES, T. Children's understanding of probability: a literature review. Nuffield Foundation. 2012, 86p. Disponível em

<http://www.nuffieldfoundation.org/sites/default/files/files/Nuffield_CuP_FULL_REP ORTv_F INAL.pdf>. Acesso em: 22. Jan. 2022.

CAMARGO, B. V. ALCESTE: um programa informático de análise quantitativa de dados textuais. In: MOREIRA, A. S. P.; CAMARGO, B. V.; JESUÍNO, J. C.; NÓBRETGA, S. M. (Org.). Perspectivas teórico-metodológicas em representações sociais. João Pessoa: Editora Universitária, 2005. p. 511-540. 
CAMARGO, B. V.; JUSTO, A. M. Tutorial para uso do software de análise textual IRaMuTeQ, 2013. Disponível em:

<http://www.iramuteq.org/documentation/fichiers/tutoriel-en-portugais> Acesso em: 10 jan. 2022.

D’AMORE, B. Elementos de Didática da Matemática. Tradução Maria Cristina Bonomi. São Paulo: Livraria da Física, 2007.

GAL, I. Towards probability literacy for all citizens: Building blocks and instructional dilemmas. In: Exploring probability in school. Springer US, 2005. p. 39-63.

KONOLD, C. Understanding students' beliefs about probability. In: VON GLASERSFELD, E. (Ed.). Radical constructivism in mathematics education. Dordrecht: Kluwer Academic Publishers, 1991. p. 139-156.

LAHLOU, S. Text Mining Methods: An answer to Chartier and Meunier. Papers on Social Representations, v. 20, n. 38, p. 1-7, 2012.

LEBLANC, J.-M. Proposition de protocole pour l'analyse des données textuelles: Pour une démarche expérimentale en lexicométrie. Nouvelles perspectives en sciences sociales (NPSS), v. 11, n. 1, p. 25-63, 2015.

MARCHAND, P.; RATINAUD, P. L'analyse de similitude appliqueé aux corpus textueles: les primaires socialistes pour l'election présidentielle française. In: Journées internationales d'Analyse statistique des Données Textuelles, JADT, 11., 2012. Actes... Liége, Belgique, 2012. p. 687-699.

MAROCCI, L. M.; NACARATO, A. M. Um ambiente de aprendizagem baseado na resolução de problemas: a possibilidade de circulação de significados sobre a Probabilidade por meio da linguagem. Educação Matemática Pesquisa, São Paulo, v. 15, n. 1, p. 101-123, 2013.

MORGADO, A. C; PITOMBEIRA, J. C; CARVALHO, P. C. P.; FERNANDEZ, P. Análise combinatória e probabilidade. Rio de Janeiro: SBM, 2004.

MUTOMBO, E. A bird's-eye view on the EC environmental policy framing: Ten years of Impact assessment at the commission. In: International Conference on Public Policy, 1., 2013, Grenoble. Anais... Grenoble: ICPP, 2013. Disponível em:

<http://www.icpublicpolicy.org/IMG/pdf/panel17_s1_mutombo.pdf>. Acesso em: 10 dez. 2020.

NASCIMENTO-SCHULZE, C. M.; CAMARGO, B. V. Psicologia social, representações sociais e métodos. Temas de Psicologia. Ribeirão Preto, v. 8, n. 3, p. 287-299, 2000.

NCTM. National Council of Teachers of Mathematics. Principios y estándares para la educación matemática. Traducción de Castellana. Sevilla: Sociedad Andaluza de Educación Matemática Thales, 2003. 
OLIVEIRA JÚNIOR, A. P. de. et al. A apreensão do conceito de experimento aleatório: resolução de problemas e jogo pedagógico. Caminhos da Educação Matemática em Revista/Online, Sergipe, v. 9, n. 2, p. 238-257, 2019.

OLIVEIRA, L. F. R. Tutorial (básico) de utilização do IRaMuTeQ. [Goiânia]: Universidade Federal de Goiás, 2015. Disponivel em:

<https://files.cercomp.ufg.br/weby/up/771/o/Tutorial_-_Revis\%C3\%A3o.pdf>. Acesso em: 14 jan. 2022.

REINERT, M. Alceste une méthodologie d'analyse des données textuel les e tune application: Aurelia de Gerard de Nerval. Bulletin of Sociological Methodology, v. 26, n. 1, p. 24-54, 1990.

SALDANHA, L.; LIU, Y. (2014). Challenges of developing coherent probabilistic reasoning: rethinking randomness and probability from a stochastic perspective. In: CHERNOFF, E. J.; SRIRAMAN, B. (Eds.). Probabilistic thinking: presenting plural perspectives. Dordrecht: Springer, 2014. P. 367-396.

SALMERÓN, E. H. El lenguaje del azar en alumnos de Educación Secundaria Obligatoria. 2015. 86 f. Dissertação (Máster en Didáctica de la Matemática) Departamento de Didáctica de la Matemática de la Universidad de Granada, Granada, España, 2015.

SANTOS, V. et al. IRaMuTeQ nas pesquisas qualitativas brasileiras da área da saúde: scoping review. In: Congresso Ibero-Americano em Investigação Qualitativa, 6., 2017. Anais... Salamanca (Espanha), 2017. p. 392-401.

SHUARD, H.; ROTHERY, A. Children Reading Mathematics. Londres: Murray, 1984.

VÁSQUEZ, C. A. O. Evaluación de los conocimientos didáctico-matemáticos para la enseñanza de la probabilidade de los profesores de educación primaria em activo. 2014. 56f. Tesis (Doctoral em Educación) - Programa de Doctorado em Educación, Universitat de Girona, Espanha, 2014.

VÁSQUEZ, C. O. Surgimiento del lenguaje probabilístico en el aula de educación primaria. REnCiMa, São Paulo, v. 9, n. 2, p. 374-389, 2018.

VÁSQUEZ, C. O.; ALSINA, A. Lenguaje probabilístico: un camino para el desarrollo de la alfabetización probabilística. Un estudio de caso en el aula de Educación Primaria. Bolema, Rio Claro (SP), v. 31, n. 57, p. 454-478, abr. 2017. 